\title{
Automatic colon polyp detection using Convolutional Encoder-Decoder model
}

\author{
Ornela Bardhi ${ }^{1}$, IEEE Member, Daniel Sierra-Sosa ${ }^{2}$, Begonya Garcia-Zapirain ${ }^{1}$, Adel Elmaghraby ${ }^{2}$ \\ ${ }^{1}$ eVIDA Lab, University of Deusto, Bilbao, Spain \\ ${ }^{2}$ Computer Engineering and Computer Science Department, University of Louisville, Louisville, KY 40292, USA
}

\begin{abstract}
Colorectal cancer is a leading cause of cancer deaths, estimated 696 thousand worldwide. Recent years have seen an increase of deep learning techniques and algorithms being used to detect colon polyps. In this work we address colon polyp detection using Convolutional Neural Networks (CNNs) combined with Autoencoders. We use 3 publicly available databases namely: CVCColonDB, CVC-ClinicDB and ETIS-LaribPolypDB, to train the model. The results obtained in terms of accuracy are: $0.937,0.951,0.967$ for the above-mentioned databases respectively. Due to the nature of the colon polyps, diverse shapes and characteristics, there is still place for improvements.
\end{abstract}

Keywords: deep learning, colon polyp detection, convolutional encoder-decoder

\section{Introduction}

In 2012 the International Agency for Research on Cancer identified Colorectal Cancer (CRC) as the 4th highest estimated number of deaths worldwide with around 696 thousand cases [1]. Nonetheless, the major concern is the elevated incidence of this cancer (3rd place with around 1.3 million cases) and the possibility to be prevented by conducting effective screening test [2-4]. Among the used screening tests to diagnose CRC, colonoscopy is the preferred technique for both, screening and prevention. Usually, colorectal cancer begins as a growth on the inner surface of the colon known as a polyp which can later develop into cancer.

During a colonoscopy the medical personnel can identify and remove colon polyps/adenomas before they progress into colon cancer. Colonoscopy has been a successful preventative procedure and has contributed to a $30 \%$ decline in the incidence of colorectal cancer [2]. A more recent study on the impact of screening on colorectal cancer mortality and incidence shows that screening colonoscopy suggest an effect of greater than $50 \%$ reduction for CRC mortality [3]. Although colonoscopy has shown such progress in reduction of mortality and incidence of CRC, the miss rate of colon polyps remains still high. A retrospective observational study published in 2017 [4] demonstrates that among 659 patients, the miss rate of colorectal polyps was $17.24 \%$ (372 out of 2158 polyps), and $38.69 \%$ of patients (255 out of 659 patients) had at least 1 missed polyp. An unidentified polyp can lead to a late diagnosis of colon cancer, associated with a survival rate of less than $10 \%$ for metastatic colon cancer. Computer-aided polyp detection provide a tool to assist colonoscopists reduce polyp miss rates.

In the recent years researchers have been working on the use of deep learning techniques to detect colon polyps [59]. Some examples of these reported contributions include Tajbakhsh et. al. using a pre-trained deep convolutional neural network (CNN) to detect colon polyps [5], Ribeiro et. al. with a method that allows the use of small patches (subimages) to increase the size of the database as well to classify different regions in the same image [6]; and the exploration of deep learning for the automated classification of colonic polyps using different configurations for training CNNs from scratch and distinct architectures of pretrained CNNs tested on 8-HDendoscopic image databases [7]. Also, authors in [8] evaluate and analyze the use of CNNs as a general feature descriptor doing transfer learning to generate CNN's features for the colon polyp classification; and developed a convolutional neural network to detect and classify two types of colon polyps, hyperplastic and adenomatous polyps by transferring different layers of low-level CNN features learned from two online nonmedical databases [9].

Our approach is based on a convolutional neural network combined with autoencoders. We tested the model on three colon databases, CVC-ColonDB [10], CVC-ClinicDB [11] and ETIS-LaribPolypDB [12] which due to its usage are now standard in the development of polyp detection techniques. These databases are open to the public for research purposes.

\section{MATERIALS AND METHODS}

\section{A. Materials}

In our study we used 3 different polyp image datasets, CVCColonDB, CVC-ClinicDB and ETIS-LaribPolypDB. CVCColonDB is the first colon polyp dataset made open, it consists of 380 images. CVC-ClinicDB consists of 612 images and ETIS-LaribPolypDB has 196 images, Table 1. Each dataset has the original images and the ground truth 
for each of them. Polyps have variations of shapes and textures as depicted in Figure 1. Some of them have the same texture as the colon and grow horizontally, leading to polyp misdetections.

Table 1. Databases included in the study

\begin{tabular}{|l|r|}
\hline Dataset & Images \\
\hline CVC-ColonDB [10] & 380 \\
\hline CVC-ClinicDB [11] & 612 \\
\hline ETIS-LaribPolypDB [12] & 196 \\
\hline
\end{tabular}

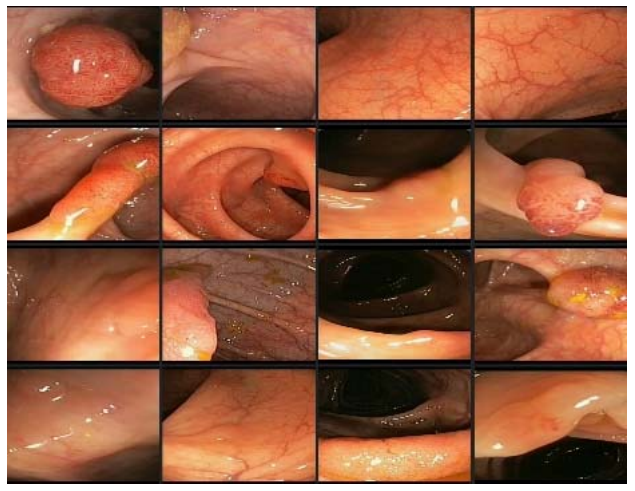

Figure 1. Different shapes and textures of colon polyps taken from colonoscopy videos ${ }^{1}$

\section{B. Methods}

We used TensorFlow library [13] for training the convolutional encoder-decoder model, which is the
TensorFlow implementation of the SegNet architecture [14], a model firstly implemented using another deep learning framework, Caffe. All training was performed using TensorFlow 1.3.0 on CUDA 8 and NVIDIA Titan X GPU. The architecture of the CNN-Autoencoder algorithm is depicted in Figure 2. The first part represents the encoder and the second part the decoder. The encoder in such models, in many cases, has a structure similar to some image classification neural networks. Layers in the decoder are the inversed layers used in the encoder (e.g. for each convolution in the encoder part, there is the deconvolution layer in the decoder part; for the max_pool, some form of "demax_pool").

In the encoder part, we use three similar "modules", each consisting of convolution layer with stride 2 followed by convolution layer with stride 1 and no-overlapping max_pool with kernel 2 . In the decoder section, each layer in the encoder contains its "counter-part". The network output dimension is equal to the input dimension:

- for no-shrinking convolution layer use the same layer

- for shrinking convolution layer use transposed deconvolution with same arguments

- for max_pool layer use nearest neighbor upsampling (tf.image.resize_nearest_neighbor)

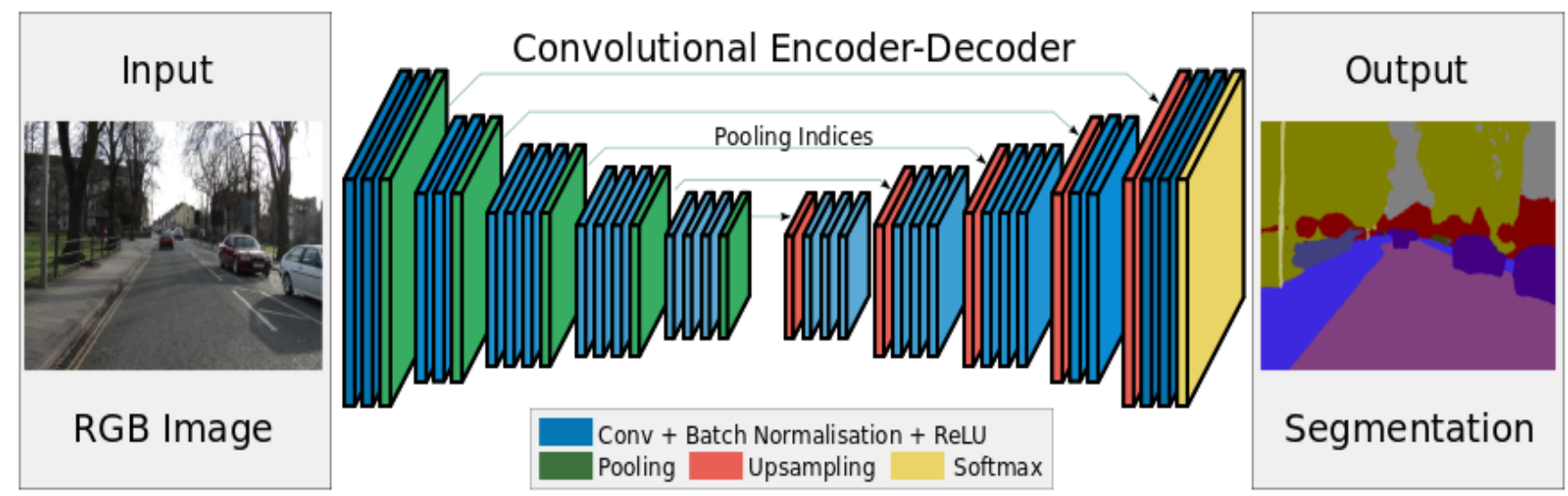

Figure 2. Convolutional encoder-decoder architecture

\footnotetext{
${ }^{1}$ These images are taken from the colon polyp database our group, eVIDA, is working. This database will be publicly available for researchers soon.
} 
Medical image databases do not have large amounts of images in them. Many researchers use image augmentations when training a model on such databases. In our model we use the Imaug library [15], an open source image augmentation library in Python. The image shown in Figure 3 is the result when some augmenters are applied to an image. Some of the image augmentations that we used are:

- Crop - crops away pixels,

- Fliplr - flips the image from left to right,

- Flipud - flips the image from up to down,

- GaussianBlur - blurs images using a gaussian kernel with size $s$,

- Dropout - sets pixels to zero with probability $\mathrm{P}$,

- AdditiveGaussianNoise - adds white/gaussian noise pixelwise to an image,

- Affine - applies affine transformation to images, such as: scaling, translating, rotating and shearing, etc.

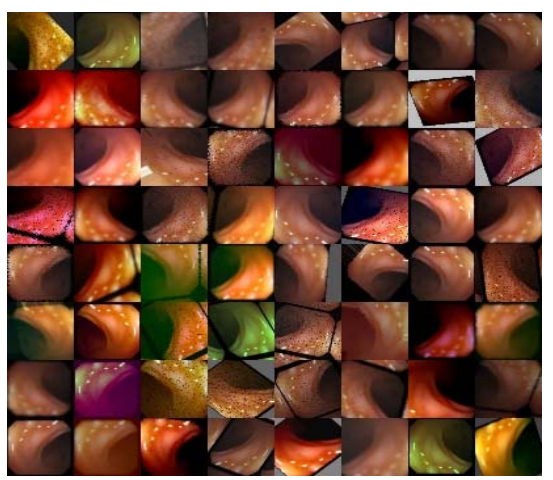

Figure 3. One image of colon polyp after applying different image augmentations

\section{Results and Discussion}

We trained our model from scratch using the selected databases. As each database has a different number of images, the time to train the model varied. Table 1 depicts, for each database, the best accuracy and in which batch that was achieved and the total training time. The best accuracy of 0.967 was achieved on ETIS-LaribPolypDB.

Table 2. Best accuracies and total time for each database

\begin{tabular}{|l|l|l|l|}
\hline Database & Best accuracy & Batch & Total time \\
\hline ETIS & 0.967 & 1300 & 1120.48 \\
\hline ClinicDB & 0.951 & 2200 & 2186.97 \\
\hline ColonDB & 0.937 & 2000 & 3659.52 \\
\hline
\end{tabular}

Polyps have different shapes and characteristics, ranging from evident protuberances in the inner surface of the colon to barely distinguishable circular shapes, this wide variation induce to errors in its recognition. In Figure 4 we present an example from the results of the proposed approach. We have selected three polyps from each of the databases (ETIS, ClinicDB and ColonDB corresponding with the left, middle and right columns from Figure 4 respectively). The first row of Figure 4 depicts the polyps' grayscale images, it should be noted that the polyp in the left column is merely visible, while the polyp in the right column is an evident protuberance easily recognizable. In the second row, the database ground truth is presented. The last row shows the obtained segments when using the CNN-Autoencoder algorithm.

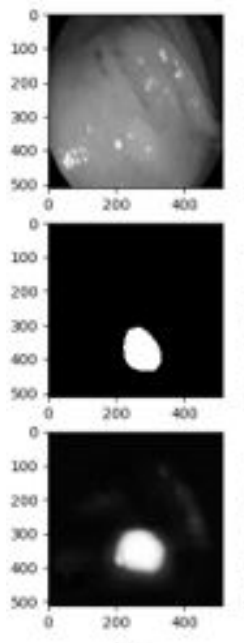

(a)

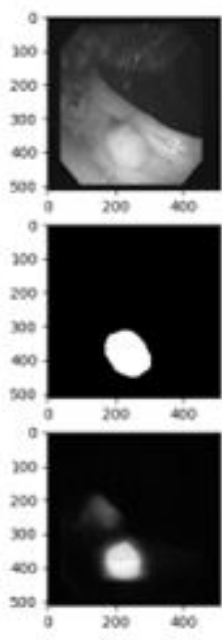

(b)

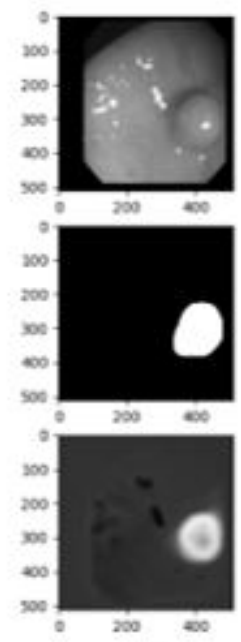

(c)
Figure 4. The results after training the convolutional encoderdecoder model on (a) ETIS-LabirPolypDB, (b) CVC-CLinicDB and (c) CVC-ColonDB database

These results are good and they correspont to the hight accuracy results we obtained. However, we have verified, visually looking at all the images we obtained, that some of the masks are not as good as expected. This is due to the shape and texture of the polyps, and the lighting conditions the image was taken. There are cases were the algorithm identifies healthy tissues as polyps, as depicted in the Figure 5.

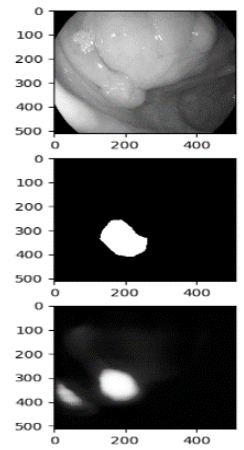

Figure 5. A false detection of a polyp 


\section{Conclusion}

The model shows promises although the results are not as expected. The same model was used on two other medical image datasets, pressure ulcers and iris, and the results were much better than with colon polyp images. One of the reasons is the difficult nature of colon polyps. Some polyps have round shapes that are very distinct form the rest of the colon, and some have the same texture as the colon making them very difficult to detect. In this paper we only tested the algorithm without making any changes. There is no image preprocessing either. We are currently making some changes on the model and we are adding four image augmentations that are not implemented yet in the Imaug library. We will train the new model with a new colon polyp database that the eVIDA group has been working in collaboration with some hospitals in the Basque Country.

\section{Acknowledgement}

Ornela Bardhi received funding from the European Union's Horizon 2020 CATCH ITN project under the Marie Sklodowska-Curie grant agreement no. 722012.

\section{References}

[1] WHO and International Agency for Research on Cancer, "Cancer Today," October 2017.

[2] D. Lieberman, "Quality and colonoscopy: a new imperative," Gastrointestinal Endoscopy, vol. 61, (3), pp. 392-394, 2005.

[3] A. G. Zauber, "The Impact of Screening on Colorectal Cancer Mortality and Incidence: Has It Really Made a Difference?" Digestive Diseases and Sciences, vol. 60, (3), pp. 681-691, 2015.

[4] J. Lee, S. W. Park, Y. S. Kim, K. J. Lee, H. Sung, P. H. Song, W. J. Yoon and J. S. Moon, "Risk factors of missed colorectal lesions after colonoscopy," Medicine, vol. 96, (27), pp. e7468, 2017.

[5] N. Tajbakhsh, J. Y. Shin, S. R. Gurudu, R. T. Hurst, C. B. Kendall, M. B. Gotway and J. Liang, "Convolutional Neural Networks for Medical Image Analysis: Full Training or Fine Tuning?" IEEE Transactions on Medical Imaging, vol. 35, (5), pp. 1299-1312, 2016.

[6] E. Ribeiro, A. Uhl and M. Hfner, "Colonic polyp classification with convolutional neural networks," in 2016 IEEE 29th International Symposium on Computer-Based Medical Systems (CBMS), 2016, pp. 253-258.

[7] E. Ribeiro, M. Hfner, G. Wimmer, T. Tamaki, J. J. W. Tischendorf, S. Yoshida, S. Tanaka and A. Uhl, "Exploring texture transfer learning for colonic polyp classification via convolutional neural networks," in 2017 IEEE 14th International Symposium on Biomedical Imaging (ISBI 2017), 2017, pp. 1044-1048.

[8] E. Ribeiro, A. Uhl, G. Wimmer and M. Häfner, "Transfer learning for colonic polyp classification using off-the-shelf CNN features," in Computer-Assisted and Robotic Endoscopy: Third International Workshop, CARE 2016, Held in Conjunction with MICCAI 2016, Athens, Greece, October 17, 2016, Revised Selected Papers, T. Peters, G. Yang, N. Navab, K. Mori, X. Luo, T. Reichl and J. McLeod, Eds. Cham: Springer International Publishing, 2017, pp. 1-13.

[9] R. Zhang, Y. Zheng, T. W. C. Mak, R. Yu, S. H. Wong, J. Y. W. Lau and C. C. Y. Poon, "Automatic Detection and Classification of Colorectal Polyps by Transferring LowLevel CNN Features from Nonmedical Domain," IEEE Journal of Biomedical and Health Informatics, vol. 21, (1), pp. 41-47, 2017.

[10] J. Bernal, J. Sánchez and F. Vilariño, "Towards automatic polyp detection with a polyp appearance model," Pattern Recognition, vol. 45, (9), pp. 3166-3182, 2012.

[11] J. Bernal, F. J. Sánchez, G. Fernández-Esparrach, D. Gil, C. Rodríguez and F. Vilariño, "WM-DOVA maps for accurate polyp highlighting in colonoscopy: Validation vs. saliency maps from physicians," Computerized Medical Imaging and Graphics, vol. 43, (Supplement C), pp. 99-111, 2015.

[12] J. Silva, A. Histace, O. Romain, X. Dray and B. Granado, "Toward embedded detection of polyps in WCE images for early diagnosis of colorectal cancer," International Journal of Computer Assisted Radiology and Surgery, vol. 9, (2), pp. 283-293, 2014.

[13] M. '. i. Abadi, P. Barham, J. Chen, Z. Chen, A. Davis, J. Dean, M. Devin, S. Ghemawat, G. Irving, M. Isard, M. Kudlur, J. Levenberg, R. Monga, S. Moore, D. G. Murray, B. Steiner, P. Tucker, V. Vasudevan, P. Warden, M. Wicke, Y. $\mathrm{Yu}$ and $\mathrm{X}$. Zheng, "TensorFlow: A system for large-scale machine learning," in 2016, pp. 265-283

[14] V. Badrinarayanan, A. Kendall and R. Cipolla, "SegNet: A Deep Convolutional Encoder-Decoder Architecture for Image Segmentation," arXiv:1511.00561v2 [cs.CV] 8 Dec 2015. Accessed October 15, 2017.

[15] Alexander Jung, "imgaug 0.2.5, " July 2017. 